\title{
CLASSICAL ANALOGOUS OF QUANTUM COSMOLOGICAL PERFECT FLUID MODELS
}

\author{
A.B. Batista|, J. C. Fabriș, S.V.B. Gonçalves and J. Tossa \\ Departamento de Física, Universidade Federal do Espírito Santo, 29060-900, Vitória, \\ Espírito Santo, Brazil
}

\begin{abstract}
Quantization in the mini-superspace of a gravity system coupled to a perfect fluid, leads to a solvable model which implies singularity free solutions through the construction of a superposition of the wavefunctions. We show that such models are equivalent to a classical system where, besides the perfect fluid, a repulsive fluid with an equation of state $p_{Q}=\rho_{Q}$ is present. This leads to speculate on the true nature of this quantization procedure. A perturbative analysis of the classical system reveals the condition for the stability of the classical system in terms of the existence of an anti-gravity phase.
\end{abstract}

PACS number(s): 04.20.Cv., 04.20.Me

The existence of an initial singularity is one of the major drawbacks of the so-called standard cosmological model. It is a general belief that such problem can be solved through the employement of a quantum theory of gravity. Indeed, near the singularity sub-Planckian scales are reached and a classical description of the Universe under this situation is not appropriate. However, there is no consistent quantum theory of gravity untill now, and in this sense the problem of the initial singularity remains of actuality. On the other hand, it is possible to construct a quantum model for the Universe as a whole, through the Wheeler-de Witt equation, based in the ADM decomposition of the gravity sector, which leads to a hamiltonian formulation of general relativity, from which a canonical quantization procedure can be applied. This gives birth to quantum cosmology[1], 8].

Quantum cosmology is not free from problems. First, it can be applied only to geometries where a foliation is possible. Moreover, the hamiltonian formalism leads to a breakdown of general covariance, and the notion of time is lost [3]. There are some recent proposals by which this notion of time can be recovered. One of these proposals is based in the coupling of the gravity sector to a perfect fluid. Using the Schutz's formulation of a perfect fluid [4], a quantization procedure is possible. The canonical momentum associated with the perfect fluid appears linearly in the Wheeler-de Witt equation, permiting

\footnotetext{
${ }^{1}$ e-mail: brasil@cce.ufes.br

${ }^{2}$ e-mail: fabris@cce.ufes.br

${ }^{3}$ e-mail: svbg@if.uff.br

${ }^{4}$ e-mail: jtoss@syfed.bj.refer.org. Permanent Adress: IMSP - Université Nationale du Bénin, Porto Novo, Bénin.
} 
to rewrite this equation in the form of a Schrödinger equation and a time coordinate associated with the matter field can be identified.

Solutions based on this approach reveal that a superposition of the wavefunctions which are solutions of the resulting Schrödinger's equation leads to a singularity-free Universe [5, 6, 7, 81. The behaviour of the scale factor may be determined in two different ways: calculating the expectation value of the scale factor, in the spirit of the many worlds's interpretation of quantum mechanics; evaluating the bohmian trajectories in the ontological formulation of quantum mechanics. The results are essentially the same in both approachs and the scale factor display a bounce, the singularity never being reached. It must be noted that even if the two procedures are technically equivalent, they are conceptually very different from each other; there are claims that from the conceptual point of view only the ontological formalism can be consistently applied to quantum cosmology[目].

The existence of a bounce indicates that there is a repulsive effect, of quantum origin, when the scale factor approachs the singularity. In this work we study more in detail such scenario. It is shown that the quantum scenario can be reproduced exactly by a very simple classical model where a repulsive fluid is added to the normal perfect fluid. It is surprising that the repulsive fluid is always the same, given by a stiff matter equation of state $p_{Q}=\rho_{Q}$, independently of the content of the normal fluid. The existence of this classical analogous of the quantum model leads us to ask questions on the true nature of the quantification in this case. Under which conditions the features of a quantum system can be exactly reproduced by a classical system? Our analysis is restricted to a perfect fluid coupled to gravity system, where the notion of time is recovered. But we sketch some considerations on other situations where gravity is coupled to matter fields.

The existence of a classical analogous of the quantum model allows us to perform a perturbative analysis establishing under which conditions the repulsive phase near singularity may be stable or not. In fact, it must be stressed that a singularity may be avoided through the violation of the strong energy condition; but, our analysis suggests that the quantum effects are due to a real anti-gravity phase, which can lead to instabilities under certain conditions.

In the perfect fluid formulation developed by Schutz, the degrees of freedom associated with the fluid are given by five scalar potentials in terms of which the four velocity is written:

$$
u_{\mu}=\frac{1}{\mu}\left(\phi_{, \nu}+\alpha \beta_{, \nu}+\theta S_{, \nu}\right)
$$

where $\mu$ is the specific enthalpy. The four velocity is subjected to the condition

$$
u^{\nu} u_{\nu}=1
$$

what enables us to express the specific enthalpy in terms of the other five potentials. The action is then given by

$$
S=\int_{M} d^{4} x \sqrt{-g} R+2 \int_{\partial M} d^{3} x \sqrt{h} h_{i j} K^{i j}+\int_{M} d^{4} x \sqrt{-g} p
$$


where $h_{i j}$ is the metric on the spatial section. The action (3) is apparently non-covariant because of the pressure term. But, in fact, the constraints intrinsic to this formalism permit to recover the covariance.

We will consider from now on the Robertson-Walker flat geometry (the spatial section must be compact in order to be consistent with the boundary conditions)

$$
d s^{2}=N^{2} d t^{2}-a(t)^{2} h_{i j} d x^{i} d x^{j} .
$$

We assume a barotropic equation of state $p=\alpha \rho$. Analyzing the conjugate momentum, and eliminating non-physical degrees of freedom, we can reduce the action (3) to [6]

$$
S=\int\left\{\dot{a} p_{a}+\dot{\epsilon} p_{\epsilon}+\dot{S} p_{S}-N H\right\}
$$

where

$$
H=-\frac{p_{a}^{2}}{24 a}-6 k a+p_{\epsilon}^{\alpha+1} a^{-3 \alpha} e^{S} .
$$

There are three cases which will interest us here: $\alpha=-1, \alpha=\frac{1}{3}$ and $\alpha=0$. The first two ones have been studied in [5, 6, 8]. Hence, first we work out in detail the third case, and after we just present the final results for the first two ones.

Following the Schutz formalism for the description of perfect fluid, and specializing it to a dust fluid, with $p=0$, we obtain the following lagrangian,

$$
L=\dot{a} p_{a}+\dot{\epsilon} p_{\epsilon}-N H
$$

where $\epsilon$ is the dust variable, with $p_{\epsilon}$ being its conjugate momemtum, and $H$ is the hamiltonian

$$
H=-\left(\frac{p_{a}^{2}}{24}+6 k a^{2}\right)+a p_{\epsilon} .
$$

Classically, this system admits, for the flat case, the well-known dust solution $a \propto t^{\frac{2}{3}}$, where $t$ is the proper time, or equivalently $a \propto \eta^{2}$, where $\eta$ is the conformal time.

Imposing the quantization rules

$$
p_{a} \rightarrow-i \frac{\partial}{\partial a} \quad, \quad p_{\epsilon} \rightarrow-i \frac{\partial}{\partial \epsilon}
$$

and considering that the hamiltonian becomes an operator which acts on the wavefunction annihilating it,

$$
\tilde{H} \Psi=0
$$

we obtain the following partial differential equation governing the behaviour of the wavefunction:

$$
\frac{1}{24} \frac{\partial^{2} \Psi}{\partial a^{2}}-i a \frac{\partial \Psi}{\partial \epsilon}=0
$$

The fact that the conjugate momentum $p_{\epsilon}$ associated to the dust fluid variable appears linearly in the hamiltonian, implies that the Wheeler-de Witt equation in the minisuperspace assumes a form similar to the Schrödinger equation with $\epsilon$ playing the role of time. Perfoming the redefinition [9]

$$
a=\frac{R}{\sqrt{12}} \quad, \quad \epsilon=\frac{t}{\sqrt{12}} .
$$


we end up with the equation

$$
\frac{1}{2} \frac{\partial^{2} \Psi}{\partial R^{2}}=i R \frac{\partial \Psi}{\partial t}
$$

We solve equation (13) using the method of separation of variables. It leads to the following decomposition of $\Psi(R, t)$ :

$$
\Psi(R, t)=\xi(R) e^{i E t}
$$

where $E$ is a (positive) constant, and $\xi(R)$ obeys the equation

$$
\xi^{\prime \prime}+2 R E \xi=0
$$

The prime means derivative with respect to $R$. The solution for (15) is under the form of Bessel functions:

$$
\xi(R)=\sqrt{R}\left(c_{1} J_{1 / 3}\left(\beta R^{3 / 2}\right)+c_{2} J_{-1 / 3}\left(\beta R^{3 / 2}\right)\right)
$$

with $\beta=\sqrt{\frac{8 E}{9}}$. The condition that the hamiltonian operator is self-adjoint, leads to two possible boundary conditions:

$$
\xi(0)=0 \quad \text { or } \quad \xi^{\prime}(0)=0 \quad .
$$

The final results is insensitive to which boundary condition we employ. Hence, we will work with the first one, but all results are essentially recovered if the second condition is used.

The general solution is a superposition of (16). In order to have analytical expressions, we will use the following superposition:

$$
\Psi(R, t)=\sqrt{R} \int_{0}^{\infty} \beta^{4 / 3} e^{-\alpha \beta} e^{i \frac{9}{8} \beta^{2} t} J_{1 / 3}\left(\beta R^{3 / 2}\right) d \beta
$$

Its solution is [10]

$$
\Psi(R, t)=\frac{R}{(2 A)^{4 / 3}} e^{\frac{-R^{3}}{4 A}}
$$

where $A=\alpha-i \frac{9}{8} t$.

It may be asked which predictions such model make for the behaviour of the scale factor. Using the many world's interpretation, this mounts to evaluate the expectation value of the scale factor. It must be stressed that essentially the same result is achieved by calculating the bohmian trajectories [2]. The measure employed in the expression is imposed again by the self-adjoint conditon, and the expression for the expectation value reads:

$$
\sqrt{12} a(t)=<R>=\frac{\int_{0}^{\infty} R^{2} \Psi(R, t)^{*} \Psi(R, t) d R}{\int_{0}^{\infty} R \Psi(R, t)^{*} \Psi(R, t) d R} .
$$

Using (19), the expectation value for the scale factor can be calculated and the final result is

$$
a(t)=\frac{1}{\sqrt{12}} \frac{2}{\alpha}^{1 / 3} \frac{\Gamma(5 / 3)}{\Gamma(4 / 3)}\left(\alpha^{2}+\frac{81}{64} t^{2}\right)^{1 / 3} .
$$


Note that the classical behaviour is recovered for $t \rightarrow \infty$. But, in general, the quantum model predicts a non-singular model exhibiting a bounce: when the singularity is approached, quantum effects leads to a repulsive effect, which leads to a regular transition from a contracting to an expanding phase.

A dynamical vacuum and a radiative fluid can be analyzed through the same lines as before. These problem were treated in [5, 6, 8] and we just present the final results.

The dynamical vacuum is realized through the equation of state $p=-\rho$. Finding the solutions of the corresponding Wheeler-de Witt equation, evaluating the expectation value of the scale factor, it results

$$
a(T)=\frac{\Gamma(4 / 3)}{\Gamma(7 / 6)}\left[\frac{64 \alpha^{2}+9 T^{2}}{8 \alpha}\right]^{1 / 6} .
$$

Asymptotically the classical solution for a cosmological constant is recovered if we choose, in the classical equations of motion, the time gauge $N=a^{-3}$ (the identification of the time coordinates can be justified rigorously [6]). In terms of the proper time, the solution (22) can be rewritten as

$$
a(t)=\frac{\Gamma(4 / 3)}{\Gamma(7 / 6)} \sqrt{8 \alpha}\left\{\cosh \left[\frac{3}{\sqrt{8 \alpha}}\left(\frac{\Gamma(4 / 3)}{\Gamma(7 / 6)}\right)^{3} t\right]\right\}^{1 / 3} .
$$

For the radiative case, the wavefunctions can be also determined through the same procedure and the wave packet constructed. The scale factor expectation value is given by

$$
a(\eta)=\frac{1}{12} \sqrt{\frac{2}{\pi \sigma}} \sqrt{\sigma^{2} \eta^{2}+(6-p \eta)^{2}},
$$

where $\eta$ is the conformal time, $p$ and $\sigma$ being real parameters. Again, this solution represents a non-singular eternal Universe which coincides asymptotically with the classical radiative solution $a \propto \eta$.

It must be stressed that in all cases, the classical behaviour is recovered for large values of the proper time. Also, both solutions are singularity free, with a bounce. Near the bounce repulsive effects appear which, in the ontological formulation, are connected with the quantum potential which corrects the classical equations of motion.

A general feature of the quantum models developped previously is the appearence of a repulsive phase for small values of the scale factor, leading to the avoidance of the singularity. In [11], it was shown that a repulsive gravity single fluid model can lead to consistent cosmological models if the curvature is negative; however, its stability is not assured in the absence of ordinary (attractive) matter. Another way of implementing a repulsive phase in classical cosmology is to consider two fluids, one that acts attractively, and the other that acts repulsively. In this case, we may have consistent solutions with flat spatial section. It is desirable that the repulsive fluid dominates for small values of the scale factor, whereas the attractive fluid dominates for large values of the scale factor. Hence, in the general, considering just the flat spatial section, we may obtain possible consistent models from

$$
3\left(\frac{\dot{a}}{a}\right)^{2}=8 \pi G\left(\rho_{M}-\rho_{Q}\right)=\frac{C_{1}}{a^{m}}-\frac{C_{2}}{a^{n}},
$$


where $p_{M}=\alpha_{M} \rho_{M}, p_{Q}=\alpha_{Q} \rho_{Q}, m=3\left(1+\alpha_{M}\right)$ and $n=3\left(1+\alpha_{Q}\right)$. The subscripts $M$ and $Q$ stand for "normal" matter component and for "quantum" repulsive component.

Ordinarilly, normal matter may be a cosmological constant, dust or a radiative fluid, corresponding to $\alpha_{M}=-1,0, \frac{1}{3}$, respectivelly. Since it is desirable that the repulsive component dominates at small values of $a$, then $\alpha_{Q}>\frac{1}{3}$. We choose then a repulsive stiff matter fluid $\alpha_{Q}=1$, what leads to $n=6$. Hence, we will solve the equation (25) with $n=6$ and $m=0,3$ and 4 . The solutions are the following:

$$
\begin{aligned}
& \alpha_{M}=-1, \alpha_{Q}=1: \quad a(t)=\left(\frac{C_{2}}{C_{1}}\right)^{1 / 6} \cosh ^{1 / 3} 3 \sqrt{C_{1}} t ; \\
& \alpha_{M}=0, \alpha_{Q}=1: \quad a(t)=\left(\frac{C_{2}}{C_{1}}\right)^{1 / 3}\left[\frac{9}{4} \frac{C_{1}}{C_{2}} t^{2}+1\right]^{1 / 3} \\
& \alpha_{M}=1 / 3, \alpha_{Q}=1: \quad a(\eta)=\sqrt{\frac{C_{2}}{C_{1}}}\left[\frac{C_{1}^{2}}{C_{2}} \eta^{2}+1\right]^{1 / 2}
\end{aligned}
$$

The comparison of the above solutions with those obtained through the construction of a superpositon of the wavefunctions resulting from the Wheeler-de Witt equation in the mini-superspace with only the ordinary perfect fluid, reveals that they are the same. Hence, wave packets constructed from a quantum model where, besides the scale factor, there is a perfect fluid matter degree of freedom (which leads to a time coordinate) are equivalent to a classical model where gravity is coupled to the same perfect fluid plus a repulsive fluid with a stiff matter equation of state $p_{Q}=\rho_{Q}$. It is really surprising that the repulsive fluid, in the classical model, is the same irrespective of the normal fluid employed in the quantum model.

This equivalence of the quantum model with a classical system different from that used in quantification process makes us to express our doubts on the true nature of the quantization scheme for this case. The question of reproducing the classical equations of motion from the quantum ones appears already in the ordinary quantum mechanics, and they are expressed in the so-called Ehrenfest's theorem. According to this theorem, the center of the wave packet may follow a classical trajectory under certain conditions. Explicitly, taking the expectation values of the Heisenberg's equations for the position and momentum operators for a particle of mass $m$ in a potential $V(\vec{r})$, we find [12]

$$
\begin{aligned}
& <\dot{\vec{r}}>=\frac{\vec{p}}{m}, \\
& <\dot{\vec{p}}>=-<\nabla V(\vec{r})>.
\end{aligned}
$$

These relations coincide with the classical one only if $\langle\nabla V(\vec{r})\rangle=\nabla V(<\vec{r}\rangle)$. This happens only for very special forms of the potential, the harmonic oscillator being an example. Only in these special cases, we may say that the center of the wave packet follows a classical trajectory.

However, the situation discussed here is somehow different from that analyzed in the Ehrenfest's theorem. In fact the expectation value of the scale factor of a quantum model derived from gravity and a perfect fluid of attractive nature is reproduced by a classical model where another fluid, of repulsive nature, appears. This seems somehow mysterious. 
Some insights into what is happening in this case may come from the employement of the ontological interpretation of quantum mechanics. In this case, the problem of time is solved in any situation (not only when a matter field is present). In fact, the ontological interpretation predicts that the system follows a real trajectory given by the equations

$$
p_{q}=S_{, q},
$$

where the subscript $q$ designates one of the degrees of freedom of the system, and $S$ is the phase of the wavefunction, which is written as $\Psi=R e^{i S}, R$ and $S$ being real functions. The equation of motion (31) is governed not only by a classical potential $V$ but also by a quantum potential $V_{Q}=\frac{\nabla^{2} R}{R}$.

These considerations suggest that the quantum potential has, at least in the case of the quantization of perfect fluid systems, a very clear behaviour which can be classically reproduced by a repulsive stiff matter fluid. However, we must stress that even in this case we must find first the wavefunction, through the Wheeler-de Witt equation, determining than its phase, from which the bohmian trajectories is computed. In the quantum models studied previously, it is not possible, in principle, to identify a classical and quantum potential in terms of the scale factor from the begining. Moreover, even if this would be possible, the classical analogous we have determined are completely independent of the Wheeler-de Witt equation.

Since we have a classical analogous of the bounce models determined through the Wheeler-de Witt equation, we can investigate if the repulsive effect leading to the avoidance of the singularity may spoil the stability of the model. First of all we define what we understand here by instability. A cosmological model is considered unstable if the perturbative variables diverge when all background quantities are finite. Here, we will consider a weaker condition: the model is unstable if the perturbed quantities takes very large values in comparison with the background quantities, even if they are not divergent. This is due to the fact that, if this happens, the hypothesis of homogeneity and isotropy, employed in the definition of the background, are compromised.

Let us consider our non singular classical system. It can be written as

$$
\begin{aligned}
R_{\mu \nu} & =8 \pi G\left[\stackrel{M}{T}_{\mu \nu}-\frac{1}{2} g_{\mu \nu} \stackrel{M}{T}\right]-8 \pi G\left[\stackrel{Q}{T}_{\mu \nu}-\frac{1}{2} g_{\mu \nu} \stackrel{Q}{T}\right], \\
\stackrel{M}{T}^{\mu \nu} ; \mu & =0 \\
\stackrel{Q}{T}_{; \mu}^{\mu \nu} & =0 .
\end{aligned}
$$

We perturb these equations in the usual way, introducing the quantities $g_{\mu \nu} \stackrel{0}{=}{ }_{\mu \nu}+h_{\mu \nu}$, $\rho_{M}=\stackrel{0}{\rho} \rho_{M}+\delta \rho_{M}, \rho_{Q} \stackrel{0}{=} \rho_{Q}+\delta \rho_{Q}$. The computation of the perturbed equations is quite standard [13], and we just present the final equations, at linear level:

$$
\begin{aligned}
\ddot{h}+2 \frac{\dot{a}}{a} \dot{h}=\frac{1}{\alpha_{M}-\alpha_{Q}}[ & -\left(1+3 \alpha_{M}\right)\left(2 \frac{\ddot{a}}{a}+\left(1+3 \alpha_{Q}\right) \frac{\dot{a}^{2}}{a^{2}}\right) \Delta_{M} \\
+ & \left.\left(1+3 \alpha_{Q}\right)\left(2 \frac{\ddot{a}}{a}+\left(1+3 \alpha_{M}\right) \frac{\dot{a}^{2}}{a^{2}}\right) \Delta_{Q}\right],
\end{aligned}
$$




$$
\begin{aligned}
\dot{\Delta}_{M}+\left(1+\alpha_{M}\right)\left(\Psi-\frac{\dot{h}}{2}\right) & =0 \\
\left(1+\alpha_{M}\right)\left[\dot{\Psi}+\left(2-3 \alpha_{M}\right) \frac{\dot{a}}{a} \Psi\right]-\frac{n^{2}}{a^{2}} \alpha_{M} \Delta_{M} & =0 \\
\dot{\Delta}_{Q}+\left(1+\alpha_{Q}\right)\left(\theta-\frac{\dot{h}}{2}\right) & =0, \\
\left(1+\alpha_{Q}\right)\left[\dot{\theta}+\left(2-3 \alpha_{Q}\right) \frac{\dot{a}}{a} \theta\right]-\frac{n^{2}}{a^{2}} \alpha_{Q} \Delta_{Q} & =0 .
\end{aligned}
$$

In these expressions, we have used the following definitions: $h=\frac{h_{k k}}{a^{2}}, \Delta_{M}=\frac{\delta \rho_{M}}{\rho_{M}}, \Delta_{Q}=$ $\frac{\delta \rho_{q}}{\delta \rho}, \Psi=\delta u_{M}^{i}, \theta=\delta u_{Q}^{i}$, where $\delta u_{M}^{i}$ and $\delta u_{Q}^{i}$ are the perturbations on the four velocity of the normal and repulsive fluid respectively.

The perturbed equations presented above do not seem to admit analytical solutions for the background solutions (26,27,28). Hence, we are obliged to perform a numerical integration. In figures 1, 2 and 3 we display the evolution of density perturbations for the exotic fluid for the cosmological constant, dust and radiative cases respectively, in the long wavelength limit $n \rightarrow 0$. The other perturbed quantities exhibits essentially the same features.

From the numerical study performed we can expose the following conclusions. When the cosmological constant is coupled to the repulsive fluid, the bounce model is unstable. Approaching the minimum of the scale factor (which in all three cases occurs in the origin), the perturbations diverge. However, when the dust or radiative fluid is coupled to the repulsive fluid, the perturbations behave regularly during all the evolution of the Universe. Hence, these models are stable. The different behaviours for the cosmological constant and the other cases may be easily understood. Indeed, in [11] a stability study was performed for the same repulsive fluid (represented there by a free scalar field); the curvature was taken to be negative. Instabilities were found in the absence of ordinary matter. What there is in common between the analysis made in [11 and the present cosmological constant plus repulsive fluid model is that when the normal fluid decouples from the repulsive fluid, the repulsive fluid becomes the only one to contribute to the right hand side of (32): it drives an unstable behaviour. In the other cases (dust and radiation), both fluids appear in the right hand side of (32), and hence the perturbations of the normal and repulsive fluid do not decouple; their interaction stabilizes the model.

In spite of the fact that we display the results for $n=0$ only, for other values of $n$ the features are very similar. For very large values of $n$, the perturbations exhibit strong oscillations, and they become divergent near the minimum of the scale factor for the cosmological constant case. Of course, we have studied the stability of the classical analogous of the quantum model. But, this study leads to some insights to what happens in the original framework.

The main point of the present work is that the quantum model, derived from the Wheeler-de Witt equation for gravity plus perfect fluid through the Schutz's formalism, 
has a classical analogous. In this classical analogous system, the perfect fluid is coupled to another perfect fluid, with a fixed equation of state $p_{Q}=\rho_{Q}$ which appears with a "wrong" sign for the gravitational coupling. The existence of this classical analogous for all equations of state of the normal fluid studied in this work, rises doubts about the true quantum nature of the original quantum cosmological scenario. It is not clear to us how to solve this doubts for the moment. But, the existence of a classical system reproducing different quantum models may indicate that the quantization of a gravity system in the mini-superspace may be not a real quantization and a more carefull analysis of this problem is deserved.

In the analysis performed previously, it has been considered a specific superposition of the solutions of the Wheeler-de Witt equation. Since these solutions are not square integrable, a superposition of them is in fact a necessity in those models. It can be argued that other types of superpositions are possible which may not be in agreement with the classical analogous treated here; all the richness of the original quantum model would not be reproduced by the classical model. However, the superposition procedure must agree with physical requirements as, for example, the localization of the wave packet, what is the case of the preceding examples. We may guess that other possible superpositions, satisfying the same physical requirements, will lead to essentially the same results.

A perturbative study was performed in the classical model. It can happens that a bounce model, where the avoidance of the singularity is obtained through an anti-gravity phase, may not be a stable model. We have verified that when the normal fluid decouples from the other perturbed equations, in such a way that the metric perturbation are coupled to the repulsive fluid only, the background model is unstable. Otherwise, we can obtain stable singularity-free models with an anti-gravity phase.

We must stress that the classical analogous model reveals that the "quantum effects" exhibit an anti-gravity behaviour. That is, the singularity is avoided with conditions much more stronger than the simple violation of the strong energy condition, as it happens in many others singularity-free models [14]. It must also be emphasized that all considerations have been done for a perfect fluid quantum model. It should be important to verify if the correspondance found here remains when gravity are coupled to matter fields. In [15] the case of a free scalar field was analyzed. However, a free scalar field is equivalent to stiff matter. Consequently, the classical analogous (if still valid for this case) would contain two kinds of stiff matter, an attractive one and a repulsive one. Perhaps, the strange behaviour founded in [15], with the quantum phase being recovered for large values of the scale factor, is due to this fact. This specific case deserves to be analyzed.

Acknowledgements: We have benefited of many enlightfull discussions with N. PintoNeto and Nivaldo Lemos. A.B.B., J.C.F. and S.V.B.G. thank CNPq (Brazil) for financial support. J.T. thanks CAPES (Brazil) for financial support and the Department of Physics of the Universidade Federal do Espírito Santo for hospitality.

\section{References}


[1] J.A. Halliwell, in Quantum cosmology and baby Universes, edited by S. Coleman, J.B. Hartle, T. Piran and S. Weinberg, World Scientific, Singapore (1991);

[2] N. Pinto Neto, Cosmology and Gravitation II, Edited by M. Novello, 229(1996);

[3] C.J. Isham, Canonical quantum gravity and the problem of time, gr-qc/9210011;

[4] B.F. Schutz, Phys. Rev. D2, 2762(1970); D4, 3559(1971);

[5] N.A. Lemos, J. Math. Phys. 37, 1449(1996);

[6] F.G. Alvarenga and N. A. Lemos, Gen. Rel. Grav. 30, 681(1998);

[7] M.J. Gotay and J. Demaret, Phys. Rev. D28, 2402(1983);

[8] J. Acacio de Barros, N. Pinto-Neto and M. A. Sagioro-Leal, Phys.Lett. A241, 229(1998);

[9] F.G. Alvarenga and N. A. Lemos, Gen. Rel. Grav. 31, 1743(1999);

[10] I.S. Gradshteyn e I.M. Rizhik, Tables of integrals, series and products, Academic Press, New York (1980);

[11] A.B. Batista, J.C. Fabris and S.V.B. Gonçalves, On the consistency of a repulsive gravity phase in the early Universe, gr-qc/0009040;

[12] C. Cohen-Tannoudji, B. Diu and F. Laloë, Quantum mechanics, volume 1, Wiley(1977);

[13] S. Weinberg, Gravitation and cosmology, Wiley, New York (1972);

[14] C.P. Constantinidis, J.C. Fabris, R.G. Furtado and M. Picco, Phys.Rev. D61, 043503(2000);

[15] R. Colistete Jr., J.C. Fabris and N. Pinto Neto, Phys.Rev. D57, 4707(1998). 


\section{Figure captions}

Figure 1: Behaviour of $\Delta_{Q}(t)$ for $n=0$ with cosmological constant.

Figure 2: Behaviour of $\Delta_{Q}(t)$ for $n=0$ with a dust fluid.

Figure 3: Behaviour of $\Delta_{Q}(t)$ for $n=0$ with a radiative fluid. 